\title{
17. EXTRAGALACTIC X-RAY SOURCES
}

\author{
G. R. BURBIDGE \\ University of California, San Diego, Dept. of Physics, \\ La Jolla, Calif. 92037, U.S.A.
}

\begin{abstract}
A review is given of the physical properties of extragalactic X-ray sources. The generation of X-rays as thermal bremsstrahlung, by the synchrotron mechanism or by Compton Scattering, is discussed. It is shown that each may be important depending on the circumstances. Only more detailed observations will enable us to decide which process dominates in any given source.
\end{abstract}

\section{Introduction}

Balloon and rocket observations of celestial X-ray sources have been made for the last decade, and in the last two years satellite observations have been under way. The most extensive catalogue of $\mathrm{X}$-ray sources so far available is that made by the American Science and Engineering group based on observations with the UHURU satellite which was launched in December 1970. While the sky has not been completely surveyed by this satellite, the results obtained, combined with those which had been made previously, give a fairly good preliminary idea of the level of activity in the $\mathrm{X}$-ray Universe.

In this paper I shall discuss only those X-ray sources which have either been tentatively identified with known extragalactic optical or radio sources.

The X-ray astronomers have begun their identifications in a period when many extragalactic objects with remarkable energetic properties in radio, optical and infrared wavelengths have been found. It is therefore natural that they have attempted to see if some of these objects can be identified as X-ray sources, as well as attempting to identify some of the bright nearby galaxies and the rich clusters of galaxies as $\mathrm{X}$-ray emitters. The lists of optical objects which have been used are therefore:

(i) Catalogues of radio sources;

(ii) Lists of quasi-stellar objects;

(iii) Seyfert galaxies and other galaxies with active nuclei;

(iv) Catalogues of bright galaxies and of clusters and groups of galaxies.

The positional uncertainties in X-ray astronomy are, for the majority of sources, very large compared with those in optical and radio astronomy. In the UHURU catalogue the sizes of the $90 \%$ confidence error boxes cover a wide range of values depending on the intensity of the source and the number of times the region has been scanned. The sizes range from several square degrees to $\sim 0.01 \mathrm{sq} \mathrm{deg}$. The limit on the flux which is detectable in the $2-6 \mathrm{keV}$ range is about $5 \times 10^{-11} \mathrm{erg} \mathrm{cm}^{-2}$ $\mathrm{s}^{-1}$.

This means that the weakest sources that can currently be detected at typical extragalactic distances will have the following luminosities: 


\begin{tabular}{lrl}
\hline Object & Distance & \multicolumn{1}{l}{$L_{x}$} \\
\hline In Magellanic Clouds & $60 \mathrm{kpc}$ & $2.0 \times 10^{37} \mathrm{erg} \mathrm{s}^{-1}$ \\
M 31 & $630 \mathrm{kpc}$ & $2.2 \times 10^{39} \mathrm{erg} \mathrm{s}^{-1}$ \\
Virgo Cluster & $15 \mathrm{Mpc}$ & $1.2 \times 10^{42} \mathrm{erg} \mathrm{s}^{-1}$ \\
Source at & $100 \mathrm{Mpc}$ & $5.3 \times 10^{43} \mathrm{erg} \mathrm{s}^{-1}$ \\
Source at & $1000 \mathrm{Mpc}$ & $5.3 \times 10^{45} \mathrm{erg} \mathrm{s}^{-1}$ \\
\hline
\end{tabular}

Very little information is available so far on the X-ray spectra of the extragalactic sources. In a few cases they have been shown not to be point sources. In Table I, I give details of the extragalactic objects of various kinds which have been identified as X-ray sources.

Among the objects identified are nearby normal galaxies, two of the classical Seyfert galaxies, NGC 4151 and NGC 1275 which is associated with an extended source, one N-system 3C 390.3 and one QSO 3C 273. The remainder are identified with radio galaxies or cluster of galaxies which contain radio sources.

The types of object identified are to some extent a reflection of the classes of optical or radio objects which have been looked for. In many sources for which no identifications have been made, the uncertainty in the position is so great that many faint

TABLE I

Some identified extragalactic X-ray sources

\begin{tabular}{|c|c|c|}
\hline Object & Type & $\begin{array}{l}\text { X-ray flux (1 unit }=1.7 \times 10^{-11} \\
\left.\mathrm{erg} \mathrm{cm}^{-2} \mathrm{~s}^{-1}\right)\end{array}$ \\
\hline Large magellanic cloud & Dwarf galaxy & $\begin{array}{l}20.7,9.3,19.3,14.9 \text { (discrete } \\
\text { sources) }\end{array}$ \\
\hline Small magellanic cloud & Dwarf galaxy & 28 \\
\hline M 31 & $\mathrm{Sb}$ & $1.9 \pm 0.3$ \\
\hline NGC 5128 & Radio galaxy & $7.4 \pm 0.4$ \\
\hline NGC 4151 & Seyfert galaxy & $3.5 \pm 0.4$ \\
\hline M 87 - Virgo cluster - 3C 274 & Radio galaxy with halo & $21.7 \pm 0.3$ (extended source) \\
\hline NGC 4696 - PKS 1245-41 & Radio galaxy in cluster & $5.9 \pm 0.4$ (extended source) \\
\hline NGC 1275 - Perseus cluster - 3C 84 & Seyfert galaxy in cluster & $43.1 \pm 0.3($ extended source) \\
\hline NGC 3862 - 3C 264 - Abell 1367 & Radio source in cluster & $3.6 \pm 0.4$ \\
\hline 3C 66 - Abell 347 & Radio source in cluster & $4.2 \pm 0.6$ \\
\hline $\begin{array}{l}\text { NGC } 4874 \text { and } 4869 \text { and Coma } \\
\text { cluster, 5C } 4.85,5 C 4.81\end{array}$ & $\begin{array}{l}\text { Extended radio source in } \\
\text { cluster }\end{array}$ & $14.9 \pm 0.3$ (extended source) \\
\hline $3 C 390.3^{a}$ & N system & $3.6 \pm 0.4$ \\
\hline Cygnus A - 3C $405^{b}$ & Radio galaxy & $5.1 \pm 1.4$ \\
\hline Hercules A-3C 348 & Radio galaxy & $6.7 \pm 1.0$ \\
\hline $3 C 273$ & QSO & $4.2 \pm 0.5$ \\
\hline 3C 129-129.1 & Radio source & $5.5 \pm 0.9$ \\
\hline NB 78.26 - Abell 2256 & Radio source in cluster & $2.9 \pm 0.3$ (extended source) \\
\hline
\end{tabular}

a Identification very uncertain.

b Identification very doubtful since sources lie in a region containing many galactic X-ray sources. Much greater positional accuracy is required before this identification can be made convincing. 
galaxies lie inside the error box. Thus the possibility that $\mathrm{X}$-ray observations will turn up a new class of extragalactic objects which emit most of their power in the $\mathrm{X}$-ray region and are not detectable as radio sources, or as objects which have very active nuclei, cannot be tested until $X$-ray source positions can be measured with much higher accuracy than is presently available. We shall briefly discuss predictions along these lines later in this paper.

\section{Processes Which Generate X-Rays}

There are three processes which may give rise to powerful X-ray sources on the galactic scale. They are:

(1) Thermal bremsstrahlung,

(2) Synchrotron radiation,

(3) Compton scattering.

In the galactic sources it has been shown that at least two of these processes are operating. In objects like Sco X-1 a significant part of the flux is simply thermal radiation from a hot gas cloud (bremsstrahlung), while in the Crab nebula the recent polarization observations by Novick et al. (1972) have shown conclusively that the synchrotron process is operating in the X-ray band. However, in the extragalactic sources no definite evidence has yet been found which demonstrates that any one of the three processes dominates. Several of the arguments which will be made in what follows can be made plausible but none is conclusive.

What are the ingredients which are required for each of these processes to work:

(1) In this case what is required is a mass of gas and an energetic source which is capable of maintaining it at temperatures of $\sim 10^{7}-10^{8} \mathrm{deg}$.

(2) In this case we need large fluxes of highly relativistic electrons and fairly strong magnetic fields. If $v=10^{19} \mathrm{~Hz}$, we require that

$$
E_{x} \approx 2.5 \times 10^{12} H_{\perp}^{-1 / 2} \mathrm{eV}
$$

while the half-lives of the electrons,

$$
\tau_{1 / 2} \approx 300 H_{\perp}^{-3 / 2} \mathrm{~s} \text {. }
$$

Thus for a range of values of $H_{\perp}$ ranging from $10^{-6}$ to $10^{3} \mathrm{G}$ we find the values shown in Table II.

TABLE II

Energies and half-lives of energies giving rise to X-rays by the synchrotron process

\begin{tabular}{lll}
\hline$H_{\perp}$ (gauss) & $E_{e}(\mathrm{ev})$ & $\begin{array}{l}\tau_{1,2} \\
\text { (seconds) }\end{array}$ \\
\hline $10^{-6}$ & $2.5 \times 10^{15}$ & $3 \times 10^{11}$ \\
$10^{-3}$ & $7.5 \times 10^{13}$ & $10^{7}$ \\
1 & $2.5 \times 10^{12}$ & 300 \\
$10^{3}$ & $7.5 \times 10^{10}$ & $10^{-2}$ \\
\hline
\end{tabular}


The magnetic fields between galaxies must be very weak indeed $\left(\ll 10^{-6} \mathrm{G}\right)$, and even in extended radio sources the 'equipartition' calculations often require fields no greater than $10^{-5} \mathrm{G}$. Thus it is clear that the generation of synchrotron X-rays in extended sources containing very weak magnetic fields is not likely unless there is an excessively large flux of very high-energy electrons present. It is only reasonable to argue that synchrotron $\mathrm{X}$-rays are generated in the vicinity of a compact object (as is the case in the Crab nebula) where much stronger magnetic fields are thought to exist. Thus it is possible that X-ray sources arising in the nuclei of galaxies and QSO's are generated at least in part by the synchrotron process (Burbidge, 1970a). If it can be established that a source has a large angular size, a synchrotron origin for the X-rays is only reasonable if it can be shown that the source is made up of a large number of compact objects. When high-resolution $\mathrm{X}$-ray observations are made, fine structure associated with such objects should eventually show up. If it does not, this would be evidence against a synchrotron origin. Conclusive evidence in favor of a synchrotron origin would be the detection of linear polarization.

(3) The ingredients required to generate an X-ray source by Compton scattering are a flux of relativistic electrons and a field of low-frequency irradiation. Since it has been known for nearly twenty years that extragalactic radio sources radiate by the synchrotron process, the existence of relativistic electrons in both small and extended regions centered on galaxies and QSO's has been established. We also know that violent activity in the nuclei of galaxies frequently gives rise both to relativistic particles and a high density of optical, radio, infrared and microwave radiation (cf. Burbidge, 1970b). Thus Compton scattering may generate X-ray sources confined to the regions of high lower frequency radiation in galactic nuclei (Burbidge, 1970a).

Also the existence of a universal field of microwave radiation has been established by the observations of Penzias and Wilson (1965) and many others following them. This may or may not be primordial radiation generated in a big bang. But independent of its origin, Compton scattering of relativistic electrons in this radiation field can be expected to give rise to extended X-ray sources (Brecher and Burbidge, 1972a).

\section{Application of Theory to Identified Sources}

For the majority of the extragalactic objects listed in Table I we do not have four critical pieces of information which might enable us to narrow down the choice of emission mechanisms. These are (1) measurements of the angular extents of the sources; (2) measurements over a wide enough energy range to give a good idea of the shape of the spectrum of the source; (3) polarization measurements; (4) detection of variability which would show that the source is very small. We do know that a few of the sources are extended (Gursky et al., 1972; Kellogg, 1972), but nothing is known about the angular sizes of the remainder.

As far as (2) is concerned the very simple-minded argument which has frequently been used and will be mentioned here is that a spectrum or exponential type suggests that the radiation is emitted by an optically thin hot gas cloud, while an inverse 
power law form would indicate a synchrotron or Compton scattering origin. As is well known, these arguments are far from conclusive. If the temperature varies through a hot gas cloud, the X-ray spectrum will not be a simple exponential, while in many of the compact radio or infrared sources, the spectra which arise from the synchrotron process, do not have a simple power-law form with a single negative index.

Among the sources listed in Table I we can make the following guesses concerning the mechanism which is operating.

\section{A. MAGELlaniC ClOUdS, OUR GALAXY AND m 31}

These are presumably prototypes of normal galaxies whose X-ray emission is coming from a number of discrete sources associated with stars, probably binary systems, with a minor contribution coming from supernova remnants. The emission mechanism in the binary systems is not really established in most cases, but thermal bremsstrahlung is likely to be important. At least for our own Galaxy, the nucleus is not an important $\mathrm{X}$-ray source. Since only a comparatively small number of discrete sources in our Galaxy and the Small Magellanic Cloud emit at power levels $\sim 10^{38} \mathrm{erg} \mathrm{s}^{-1}$, and they apparently make up the bulk of the total X-ray emission from such galaxies, there may be very considerable ranges in X-ray luminosity between galaxies of similar types since the number of powerful sources may be a function of the number of binaries at the critical emitting phase, etc.

B. NGC 5128, NGC 4151, ANY COMPACT SOURCE ASSOCIATED WITH M 87, NGC 4696, NGC 1275, 3C 390.3 AND 3C 273

All of these extragalactic sources are known to have very active nuclei in optical, infrared, microwave or radio wavelengths. The data on the Seyfert nuclei in NGC 4151 and NGC 1275 and the measurements of M 87, the N-system 3C 390.3 and the QSO 3C 273 have been discussed in detail elsewhere (Burbidge, 1970b). Recently a small bright infrared nucleus has been discovered in NGC 5128 (Kunkel and Bradt, 1971), while NGC 4696 is a southern radio galaxy containing a radio source of small angular size (Ekers, 1968).

If it can be shown that the $\mathrm{X}$-ray sources arise in these active nuclei, then in principle any one of the three mechanisms previously described can be operating. Evidence for the existence of high-speed mass motions of gas in the nuclei of NGC 4151, NGC 1275 and in 3C 390.3 is available from the optical observations. Thus the X-ray emission may arise from the dissipation of kinetic energy contained in these motions. Alternatively the Compton or synchrotron processes taking place in a tiny nucleus in which the nonthermal optical and infrared flux is generated may be responsible as was originally proposed for the nucleus in M 87 (Burbidge, 1970a).

\section{EXTENDED X-RAY SOURCES ASSOCIATED WITH CLUSTERS OF GALAXIES AND RADIO SOURCES}

At present we have evidence that four of the sources listed in Table I, namely the sources in the Virgo, Perseus, Coma and Abell 2256 clusters (Kellogg, 1972), and 
the source in the cluster of which the brightest member is NGC 4696, are extended, with sizes ranging from $\sim 30^{\prime}$ to $\sim 50^{\prime}$ corresponding to linear dimensions $\sim 200-800$ kpc. Moreover a number of the other proposed identifications are with clusters of galaxies containing radio sources, though we have no size measurements for the $\mathrm{X}$-ray sources and in some cases none for the radio sources either. While Gursky et al. (1972) have stressed the correlation between extended X-ray sources and rich clusters of galaxies, studies of correlations between (a) powerful X-ray sources and clusters of galaxies, and (b) powerful X-ray sources and extended radio sources (Brecher and Burbidge, 1972b) have shown that both correlations are present and that therefore neither hypothesis can be rejected. If (a) alone were important and if a further correlation could be found between the X-ray luminosity and the dynamical state of the cluster or some other physical property of the galaxies in the cluster, it would be reasonable to conclude that the radiation is thermal bremsstrahlung from a hot gas whose energy ultimately derives from the galaxies in the cluster or it might be generated in the galaxies directly. While the sample of sources is very small, Solinger and Tucker (1972) have claimed to have found such a correlation. Namely, for three clusters, Virgo, Perseus and Coma, they find the data to be consistent with $L_{x} \propto(\Delta v)^{4}$, where $\Delta v$ is the velocity dispersion of the galaxies in the cluster. It is hard to decide how much significance should be attached to such a relation. The problem is that while the velocity dispersions in the three clusters only vary by a rather small factor, the clusters are rather different in their galactic populations and probably in their states of evolution. A discussion of the optical properties of the clusters has been given in this Symposium by Sargent (p. 184). In the Virgo cluster two subclusters of spirals and ellipticals, respectively, have been identified, the Perseus cluster contains only a comparatively small number of galaxies arranged in two chain-like structures, while the Coma cluster is a very rich cluster with a more symmetrical distribution of galaxies, and it apparently only contains ellipticals and SO galaxies. In each cluster the visible galaxies are not able to bind the system, and if it is argued that they are stable configurations very large amounts of dark material, either in the form of uncondensed gas or of condensed objects with very high massto-light ratios are required to stabilize them. While many investigators have taken this as evidence that much dark matter must be present, others have taken the view that the clusters are unstable and are expanding (cf. the discussion of Burbidge and Sargent, 1969). Thus it is not possible from these arguments based on the virial theorem to draw any certain conclusions about the amount of hot gas that may be present. However, in each case it is easily shown that only a comparatively small mass of hot gas, perhaps only a few percent of the mass of the galaxies if optimum values are taken, is required to account for the X-rays as thermal bremsstrahlung. Such gas could have been ejected from the galaxies and be heated by the motions of the galaxies.

If the correlation between extended radio sources and X-ray sources were shown to be all important, this would be a strong argument in favor of the suggestion that the X-rays are generated by Compton collisions between photons of the microwave 
background radiation and the relativistic electrons in the radio sources. This argument looks very plausible for the Coma cluster (Brecher and Burbidge, 1972a) and for other sources listed in Table I (Brecher and Burbidge, 1972b; Bridle and Feldman, 1972; Perola and Reinhardt, 1972; Costain et al., 1972).

The relationship between the power emitted by the synchrotron process and that radiated by Compton scattering is given by (Felten and Morrison, 1966)

$$
\frac{L_{r}}{L_{x}}=\frac{H^{2}}{8 \pi \varrho}\left\{\frac{20000 T_{b}}{H}\right\}^{(3-m / 2)}
$$

where $H$ is the effective magnetic field in the source, $\varrho$ is the energy density of the radiation field and $T_{b}$ is its brightness temperature, and $m$ is the electron spectral index $\left(N(E)=\mathrm{k} E^{-m}\right)$. For $m=3$ corresponding to a synchrotron or Compton radiation spectrum $I \propto v^{-1}$, this relation simply reduces to the fact that the ratio $L_{r} / L_{x}=\left(H^{2} / 8 \pi\right) / \varrho$.

Except in the case of Centaurus $A$, the extended radio source surrounding NGC 5128 from which no X-ray flux has been detected, the sensitivity limits of the X-ray detectors presently in use lead to the result that where an extended X-ray source has been identified with a known radio source, $L_{x} \gg L_{r}$.

In the simplest case, with $m=3$, and assuming that the microwave background radiation is of black body form with $T_{b} \simeq 3 \mathrm{~K}$,

$$
L_{r}=L_{x}\left(2.5 \times 10^{5} H\right)^{2} .
$$

Thus, if $L_{x} \gg L_{r}, H \ll 4 \times 10^{-6} \mathrm{G}$. If the microwave background radiation has a much higher radiation density, as has been suggested by some of the rocket observations, then the lower limit on $H$ will be raised accordingly. Calculations of the energetics of the well-known extended radio sources (Burbidge, 1959; Maltby et al., 1963) show that if the so-called equipartition condition applies, though there is no physical basis for this, the magnetic field strengths required are in general $\sim 10^{-5} \mathrm{G}$ or greater. Thus, provided that the same regions give rise to the radio emission and the X-ray emission, this argument would suggest that the magnetic field is considerably weaker than the equipartition value (perhaps $\sim 10^{-7} \mathrm{G}$ ) and the total energy in particles in the sources must be increased over the equipartition value by a factor $\left(H_{\text {eq }} / H\right)^{3 / 2}$. However, to generate $\mathrm{X}$-ray photons with energies $\sim 5 \mathrm{keV}$ from photons of the microwave background $\left(\lambda_{\max } \simeq 1 \mathrm{~mm}\right)$ requires electrons with energies $\sim 1-2$ $\mathrm{GeV}$, and in a field as weak as $10^{-7} \mathrm{G}$ such electrons will radiate synchrotron radiation of rather low frequencies, $<10 \mathrm{MHz}$. Consequently, if the magnetic fields are very weak, the electrons largely responsible for the $\mathrm{X}$-rays will have lower energies than those responsible for the radio flux in the meter and centimeter wavelength range. Thus a more detailed investigation is required to apply relation (1) precisely. We require to know the shapes of the radio and X-ray spectra over a wide spectral range and also to determine from observation whether the regions of radio and optical emission coexist. A fairly detailed model which will fit the observations has been made for the Coma cluster (Brecher and Burbidge, 1972a). 
It is well known that many of the strong radio sources are double with two fairly well defined regions of emission symmetrically placed about the optical galaxy, which may be the brightest member of a cluster of galaxies. It is possible in such cases that the X-ray emission comes from a much more extended volume than the double radio source and that the magnetic field in this more extended region is much weaker than that in the strongly radio-emitting source.

From this discussion it is clear that it is too early for us to decide whether the extended X-ray sources are due to Compton scattering or thermal radiation from a hot gas. The third possibility, that such extended emitting regions are due to X-ray emission from many powerful galaxies or other discrete objects, also cannot be excluded.

\section{NEW TYPES OF EXTRAGALACTIC OBJECTS}

In this paper we have only discussed so far extragalactic X-ray sources which have been identified with special types of optical or radio sources. We conclude by pointing out that it is clearly possible that some of the X-ray sources are of a completely new type which are not known to radiate powerfully in optical, infrared or radio wavelengths. Can we predict what kinds of objects might be found?

One possibility is that they are highly compact massive objects which generate nonthermal radiation in ultraviolet wavelengths by the synchrotron process. The prediction of the existence of such objects is a natural extension of our present knowledge of QSO's or of the nuclei of galaxies. If an intense flux of far UV radiation, say in the wavelength range $500-1500 \AA$, is generated in a small volume, Compton scattering of comparatively low-energy electrons $(E \approx 100 \mathrm{MeV})$ would lead to the generation of very powerful fluxes of X-rays in the $1-100 \mathrm{keV}$ energy range. Estimates of the flux can be made in a way similar to that calculated for the efficiency of the Compton process involving microwave radiation in the nucleus of $M 87$ (Burbidge, 1970b). Such objects need not be powerful UV emitters, and they would not have been discovered by other techniques so far. Since the nuclei of galaxies which are powerful infrared emitters may be radiating by a process involving nonthermally generated UV photons heating dust, such sources might be condensed objects in the nuclei of faint galaxies in which dust is absent.

\section{Acknowledgement}

Research in extragalactic astronomy at the University of California, San Diego, is supported by the National Science Foundation and by NASA through Grant Number NGL 05-005-004.

\section{References}

Brecher, K. and Burbidge, G. R.: 1972a, Astrophys. J. 174, 253.

Brecher, K. and Burbidge, G. R.: 1972b, Nature 237, 440.

Bridle, A. H. and Feldman, P. A.: 1972, Nature Phys. Sci. 235, 168.

Burbidge, G. R.: 1959, in R. N. Bracewell (ed.), 'Paris Symposium on Radio Astronomy', IAU Symp. 9, 541, Stanford University Press. 
Burbidge, G. R.: 1970a, Astrophys. J. Letters 159, L105.

Burbidge, G. R.: 1970b, Ann. Rev. Astror. Astrophys. 8, 369.

Burbidge, G. R. and Sargent, W. L. W.: 1969, Comm. Astrophys. Space Phys. 1, 220.

Costain, C. H., Bridle, A. H., and Feldman, P. A.: 1972, preprint.

Ekers, R. D.: 1968, Austral. J. Phys. Astrophys. Suppl., No. 6.

Felten, J. and Morrison, P.: 1966, Astrophys. J. 146, 686.

Gursky, H., Solinger, A., Kellogg, E. M., Murray, S., Tananbaum, H., Giacconi, R., and Cavaliere, A.: 1972, Astrophys. J. Letters 173, L99.

Kellogg, E. M.: 1972, this volume, p. 171.

Kunkel, W. E. and Bradt, H. V.: 1971, Astrophys. J. Letters 170, L7.

Maltby, P., Matthews, T. A., and Moffet, A. T.: 1963, Astrophys. J. 137, 153.

Novick, R., Weisskopf, M. C., Berthelsdort, R., Linke, R., and Wolff, R. S.: 1972, Astrophys. J. Letters 174, L1.

Perola, G. and Reinhardt, M.: 1972, Astron. Astrophys. 17, 432.

Penzias, A. A. and Wilson, R.: 1965, Astrophys. J. 142, 419.

Sargent, W. L. W.: 1972, this volume, p. 184.

Solinger, A. and Tucker, W.: 1972, preprint.

Weisskopf, M. C., Novick, R., Berthelsdort, R., Linke, R., and Wolff, R. S.: 1972, Bull. Am. Phys. Soc. 17, 501; 